\section{Co-Evolution of Nutrition and Immunity}

\author{
Petr Sima ${ }^{1}$, Vaclav Vetvicka ${ }^{2 *}$ and Luca Vannucci ${ }^{1}$ \\ 'Laboratory of Immunotherapy, Institute of Microbiology, Prague, Czech \\ Republic \\ ${ }^{2}$ Department of Pathology, University of Louisville, Louisville, KY, USA
}

\begin{abstract}
The influence of nutrition on immunity studies the relatively young field of biomedical sciences, nutritional immunology. Nutritional immunology, in other words, the immunology of nutrition, often also referred to as immunonutritology, is a relatively new branch of medical sciences connecting two, at first glance, quite different fields, the immunology, and the science of nutrition, the nutritology. In spite of this dissimilarity, immunonutritology has a broad impact on the health status of all layers of the population. Countless amounts of evidence indicates that non-adequate or reduced intake of nutrition was reflected not only in the classic signs of protein and energy malnutrition (wasting or marasmus, kwashiorkor, etc.), but also a serious disturbance in the failure of immune functions. On the other hand, long-lasting increased feed intake is also connected with a negative effect on immunity.
\end{abstract}

Keywords: Cytokines; History; Immunity; Nutrition

\section{History}

It is proverbial for the history of science and for medicine in particular that old knowledge and experiences are often long forgotten, only to be re-discovered. This also applies to understanding the influence of nutrition on human health. Already in antiquity it has been known that diet directly affects health. Hippocrates (5th century BC) had a completely modern attitude when he noted that "health consists of proper mixing of body juices of various kinds... and medicine this is achieved by an appropriate diet..." [1]. In this context it should be recalled that' dietetic' was a doctrine about the right way of life (including hygiene and gymnastics) and "hédiaíta" meant in ancient nations did not thought only about food. In an essay "On Ancient Medicine" (Periarchaiésiétrikés) is noted that the development of medicine depended mainly on prescribing special diets. Aulus Cornelius Celsus (35 BC-50 AD) was of the same opinion. He recommended a salt-free diet in kidney diseases, prescribed liver for therapy of night blindness, and advised people to

*Corresponding author: Vaclav Vetvicka, Department of Pathology, University of Louisville, Louisville, KY 40202, USA, Tel: +1 5028521612; Fax: +1 5028527674; E-mail: Vaclav.vetvicka@louisville.edu

Citation: Sima P, Vetvicka V, Vannucci L (2016) Co-Evolution of Nutrition and Immunity. J Food Sci Nutr 2: 008.

Received: February 24, 2016; Accepted: March 25, 2016; Published: April 11 2016 drink milk in poisoning. A physician of Marcus Aurelius emphatically pioneered the treatment of diseases by using the specific diets of Galen of Pergamum (130-201 AC) [2].

For many centuries these recommendations were almost for gotten and even in the $17^{\text {th }}$ and $18^{\text {th }}$ centuries diets and especially some of their composite components for the prevention and treatment of diseases were completely ignored. In 1754 however, J Lind, an English ship's doctor considered the founder of "naval hygiene in England", in his "A Treatise of the Scurvy" noted that scurvy (a disease caused by vitamin $\mathrm{C}$ deficiency), killed more seamen than were killed in battle [3]. On the basis of two hundred year old knowledge of Dutch people about the benefits of citrus fruits to the health of the sailors on long voyages, J Lind conducted one of the first large-scale clinical medical trials and in his next important paper "on the most effectual means of preserving the health of seamen" published three years later, proposed the eradication of scurvy from the British Navy using fresh citrus fruit and juice prepared from them [4]. In 1795, when his recommendation was definitively adopted and implemented, scurvy definitively disappeared from the British Fleet, apparently by "magic". However, we have to remember that it took 70 years for the scientific community to accept that vitamin $C$ cured scurvy.

In $1810 \mathrm{~J}$ F Menkel described atrophy of the thymus, the central organ of immunity, in which the functional subpopulations of immunocompetent cells differentiate and mature, as a result of inadequate nutrition and fasting [5]. About 35 years later, J Simon presented a detailed study on the thymus, in which he considers thymus as a "barometer of malnutrition" [6]. At the beginning and in the first half of the $20^{\text {th }}$ century, the importance of vitamins as essential nutrients, whose inadequate intake causes severe immunodeficiency, was gradually discovered and conclusively documented. Concurrently, the influence of exhaustion and malnutrition upon growth was studied [7]. Significant textbooks of nutritology issued in the late forties of the $20^{\text {th }}$ century still contain only a brief note on the relationship of nutrition on infection, where it was stated that, with the possible exception of tuberculosis there is no evidence that the interrelationship among infectious disease, nutrition and immune status is confirmed [8].

Nutritional immunology was intensively developed in the 60 s and 70 s when immunology as a separate medical discipline, completely detached from microbiology and serology. This period is now traditionally known as the "golden age of immunology". New discoveries in immunology were rewarded by several Nobel prizes and were accompanied by wide interest by laymen and specialists in the impact of individual nutritional components on the immune status of the organism. The research focused mainly on determining the optimal composition of nutrition that was used particularly in the prevention of infectious diseases, to support the treatment of ongoing disease and in convalescence. Gradually, experts began to understand that every infectious disease is accompanied by a reduction of food intake, both of which culminate in deterioration of the immune system.

In 1968, the World Health Organization published a monograph dealing with the relationship between nutrition and infection, when 
the synergistic relationship between malnutrition and infection was first introduced [9]. In the early seventies, it was definitively demonstrated that protein-calorie malnutrition is the cause of extreme involution of lymphoid tissues including the thymus. Therefore, it was known as nutritional thymectomy [10]. Its consequences are reflected in a reduction of the functional effectiveness of both cellular and humoral branches of immunity. In cellular immunity there was attenuation delayed hypersensitivity reactions, decreased number of $\mathrm{T}$ cells and phagocytic dysfunction. Effects on humoral immunity were seen as reduced production of secretory IgA and humoral antibodies with effects on their affinity, and low activity of complement components [11]. Nutrition and infections are intimately related, which is particularly important for young children who frequently suffer respiratory problems and diarrheal disease. The link between malnutrition and impaired immune functions is well described [12].

Until relatively recently, in 2003, the World Health Organization defined nutritional recommendations, which should be applied in the prevention and treatment of the most common communicable and non-communicable diseases including cancer. These recommendations were based on extensive experimental and clinical studies, which suggested that nutritional composition of dietary intake could support immune functions. Beneficial effects could be attributed to a reduction in unsaturated fatty acids, high fiber content, and the presence of biological response modifiers such as muramyl peptides, transfer factor, and particularly glucans. These benefits were particularly evident in people under situations of environmental stress, prolonged illness, radiation exposure, treatment with chemotherapeutics or immunosuppressant's $[13,14]$.

\section{Interrelationships Between Nutrition and Immunity}

Interrelationships between nutrition and immunity are considered completely interconnected [15]. It is shown that immunity is compromised in malnutrition, poor nutrition and during starvation. An abundance of food can also have adverse effects on immunity to increase susceptibility to infectious and other non-communicable diseases [16].

Trauma and stress accompanying the development of disease converge into a catabolic state, which leads to further nutritional insufficiency, which in turn worsens the course of infection. This creates a vicious circle that can interrupt treatment. It is estimated that inadequate nutrition is the cause of up to $60 \%$ non-infectious and more than $75 \%$ of infectious diseases. Syndromes accompanying nutritionally induced dysfunctional immunity are so similar to HIV induced AIDS that the conditions are described as Nutritionally Acquired Immune Deficient Syndromes (NAIDS) [5]. In developed countries it is not generally appreciated that in the Third World NAIDS affects the bulk of the population of all ages and that it is also the primary cause of morbidity and mortality.

Concomitant illness also worsens the nutritional status of an individual, and consequently also its immune capacity [17]. There are numerous infectious diseases that are accompanied by malabsorption of nutrients. They are especially bacterial, viral and protozoan enteritis, intestinal helminthiases and febrile diseases, which lead to malabsorption of proteins, important vitamins and trace elements. Virtually all infections, even if they are not accompanied by fever, cause catabolic loss. The first response to infection is caused by cytokines that are also implicated in anorexia. Cytokines and chemokines such as IL-1, IL-6, IL-8 and TNF-alpha can decrease food intake through their influence on the central nervous system. Through their induction of febrile response they also result in decreases in the concentrations of $\mathrm{Fe}, \mathrm{Cu}$, and $\mathrm{Zn}$, thus preventing their utilization by pathogenic microbes [18]. Under the influence of the increased production of IL-1 and changes of endocrine secretions the amino acids are mobilized from the skeletal muscle and used for liver gluconeogenesis, while nitrogen is removed by the urine [19].

Average protein loss in adults recalculated for normal infection are $0.6 \mathrm{~g} . \mathrm{kg}^{-1}$ per day, for the diseases accompanied by diarrhea then $0.9 \mathrm{~g} . \mathrm{kg}^{-1}$ per day, and typhoid fever and severe infections to $1.2 \mathrm{~g} \cdot \mathrm{kg}^{-1}$ per day [20]. Fever, on average, increases basal metabolic rate by $13 \%$ with an increase in body temperature every $1^{\circ} \mathrm{C}$ and a high fever by one third, whereas nitrogen and amino acids are lost through sweating [21].

In 1980 an extensive review was published, which summarized experimental and clinical studies on the effect of nutrition on immunity from the sixties. From these very important and still valid data it can be concluded that protein and energy malnutrition compromises immune function, with the greatest impact on phagocytosis and complement system. Cellular immunity is affected even more than humoral immunity. These effects exacerbate deficiency of vitamins (especially A, B6, folate, riboflavin and thiamine) and trace elements $\mathrm{Fe}, \mathrm{Zn}$, and especially Se. Certain micronutrients mentioned above also possess important antioxidant functions involved not only in regulation of immune homeostasis, but also altering the genome of the microbes and viruses [22]. For more information on the history of nutrition, see Keusch, 2003) [23].

The conclusions of the studies published from1966 to 2006 concerning nutritional support of anti-infectious immunity by means of various vitamins and multivitamin preparations, trace elements and probiotic yogurts suggest that therapeutic effects are not unambiguous and nutritional supplement (perhaps with the exception of glutamine) do not help in the treatment of certain on-going infections [24].

Another important aspect is a possibility of immunomodulation by food. In last 50 years, there has been a decrease of classical infection diseases, but an increase in immune-related disorders including allergies. In addition, formulation of specific nutritional formulation in necessary for critically-ill or old population with suppressed immunity. Such preparation contain specific amino acids such as glutamine and arginine, nucleotides, and specific fatty acids [25]. Another benefits include addition of probiotics or prebiotics, particularly fructose oligosaccharides and galastose oligosaccharides [26]. Studies using children in Sweden revealed that allergic pre-school children were less often colonized with Lactobacilli and more often with aerobic coliforms [27]. These data are relevant to the proper maturation of intestinal immunity, particularly to the balance between Th1 and Th2 lymphocytes [28]. Possible immunomodulation by food was elegantly reviewed in recent study written by Wichers [29].

\section{Conclusion}

The relations between nutrition and immunity can be summarized in four basic levels: 1) nutrition determines development of the immune system in early life stages and its efficiency in adult life; 2) inadequate intake of protein as energy as well as of some individual nutritional components affect the immunity; 3) hyper alimentation leading to excessive weight gain and obesity directly affects immunity; 
and 4) research into the interactions between nutrition and immunity is of practical importance for clinical medicine and public health.

In recent decades, immune disorders due to inadequate nutrition are also increasingly found in industrialized countries. This is through environmental pollution and stress factors surrounding the current high pace of life, as well as intake of unbalances and processed foods containing undesirable components added to enhance flavor and increase shelf-life. This is truefor the populationin industrialized countries, and more recently in emerging countries of the so called Third World, which are increasingly adopting a westernized lifestyle and dietary habits. This has paradoxically led to a spread of malnutrition through over consumption, often of empty calories, leading to excessive weight and obesity resulting in nutrient depletion that compromises immunity [30].

\section{Acknowledgement}

This work was supported by the institutional grant number RVO 61388971 .

\section{References}

1. Thorwald J (1963) Science and the Secrets of Early Medicine. Harcourt, Brace and World, New York, USA.

2. Ackerknecht EH (1973) Therapeutics from the primitives to the 20th century (with and appendix: history of dietetics). MacMillan Publishing, New York, USA.

3. Lind J (1757) A Treatise of the Scurvy in Three Parts. Containing an inquiry into the Nature, Causes and Cure of that Disease, together with a Critical and Chronological View of what has been published on the subject. (2ndedn), A Millar, London, USA.

4. Lind J (1762) An Assay On the Most Effectual Means of Preserving the Health of Seamen in the Royal Navy: containing directions proper for all those who undertake long voyages at sea, or reside in unhealthy situations; with cautions necessary for the preservation of such persons as attend the sick in fevers. D Wilson, London, UK.

5. Beisel WR (1992) History of nutritional immunology: introduction and overview. J Nutr 122: 591-596.

6. Simon J (1845) A Physiological Essay on the Thymus Gland. H Renwhaw, London, UK.

7. Jackson CM (1925) The Effects of Inanition and Malnutrition Upon Growth and Structure. P. Blakiston's Son \& Co, Philadelphia, USA.

8. Jeliffe N (1950) Textbook of Clinical Nutrition. Harper \& Brothers, New York USA.

9. Scrimshaw NS, Taylor CE, Gordon JE (1968) Interactions of nutrition and infection. Monogr Ser World Health Organ 57: 3-329.

10. Smythe PM, Brereton-Stiles GG, Grace HJ, Mafoyane A, Schonland M, et al. (1971) Thymolymphatic deficiency and depression of cell-mediated immunity in protein-calorie malnutrition. Lancet 2: 939-943.
11. Chandra RK (1999) Nutrition and immunology: from the clinic to cellular biology and back again. Proc Nutr Soc 58: 681-683.

12. Law DK, Dudrick SJ, Abdou NI (1974) The effects of protein calorie malnutrition on immune competence of the surgical patient. Surg Gynecol Obstet 139: $257-266$.

13. Torrence PF (1985) Biological Response Modifiers. Academic Press, London, UK.

14. Vetvicka V (2013) $\beta$-Glucans as Natural Biological Response Modifiers. Nova Biomedical, New York, USA.

15. Mainous MR, Deitch EA (1994) Nutrition and infection. Surg Clin North Am 74: 659-676.

16. Chandra RK (1992) Nutrition and immunology. St. John's, Newfoundland: ARTS Biomedical.

17. Scrimshaw NS, SanGiovanni JP (1997) Synergism of nutrition, infection, and immunity: an overview. Am J Clin Nutr 66: 464-477.

18. Mackowiak PA (2005) Temperature regulation and the patogenes is of fewer. In: Mandell GL, Bennet JE, Dolin R (eds.). Principles and Practice of Infection Diseases. Elsevier Churchill Livingstone, Philadelphia, USA. Pg: 703-718.

19. Keusch GT, Farthing MJ (1986) Nutrition and infection. Annu Rev Nutr 6: 131-154.

20. Powanda MC (1977) Changes in body balances of nitrogen and other key nutrients: description and underlying mechanisms. Am J Clin Nutr 30: 12541268.

21. DuBois EF (1936) Basal Metabolism in Health and Disease. Lea and Febiger, Philadelphia, USA.

22. Bhaskaram $P$ (2002) Micronutrient malnutrition, infection, and immunity: an overview. Nutr Rev 60: 40-45.

23. Keusch GT (2003) The history of nutrition: malnutrition, infection and immunity. J Nutr 133: 336-340.

24. Donabedian H (2006) Nutritional therapy and infectious diseases: a twoedged sword. Nutr J 5: 21.

25. Fernandes G (2008) Progress in nutritional immunology. Immunol Res 40: 244-261.

26. de Vrese M, Schrezenmeir J (2008) Probiotics, prebiotics, and synbiotics. Adv Biochem Eng Biotechnol 111: 1-66.

27. Björkstén B, Naaber P, Sepp E, Mikelsaar M (1999) The intestinal microflora in allergic Estonian and Swedish 2-year-old children. Clin Exp Allergy 29: 342-346.

28. Kalliomäki M, Salminen S, Poussa T, Arvilommi H, Isolauri E (2003) Probiotics and prevention of atopic disease: 4-year follow-up of a randomised placebo-controlled trial. Lancet 361: 1869-1871.

29. Wichers $H$ (2009) Immunomodulation by food: promising concept for mitigating allergic disease? Anal Bioanal Chem 395: 37-45.

30. Bengmark S (1998) Ecoimmunonutrition: a challenge for the third millennium. Nutrition 14: 563-572. 